\title{
Comparative Studies of Hydration Behaviour and Density of Pyrrolidone K-30, PEG 6000 and PEG 20000
}

\author{
Sakina Sultana, Md. Ohidullah, A. H. M. Saifuddin and Md. Saddam Hossain \\ Department of Pharmacy, Jahangirnagar University, Savar, Dhaka-1342, Bangladesh
}

Received: July 23, 2014; Accepted: January 22, 2015

\begin{abstract}
A comparative study was conducted on aqueous solutions of Pyrrolidone K-30 and Polyethylene glycols of molecular weight 6000 and 20000, respectively for investigating their hydration behaviour in terms of intrinsic viscosity and density. In the study, Pyrrolidone K-30 exhibited the intrinsic viscosity of $\sim 14 \mathrm{~g} / \mathrm{ml}$, while PEG 6000 and PEG 20000 revealed the intrinsic viscosities of $18 \mathrm{~g} / \mathrm{ml}$ and $28 \mathrm{~g} / \mathrm{ml}$, respectively at a room temperature of $298 \pm 1^{\circ} \mathrm{K}$. Thus, among these three compounds PEG 20000 exhibited the highest intrinsic viscosity which was interesting. In density measurement this compound showed a non-linear trend of change in density as concentration was varied. Such results were opposite to that observed with the remaining two compounds.
\end{abstract}

Key words: Pyrrolidone K-30, PEGs, Hydration and Density.

\section{Introduction}

Polymers constitutes an important class of compounds of industrial interest. Chemically polymers are synthetically produced high molecular weight compounds containing a number of smaller and identical repeating units known as 'monomers'. Each monomer is bonded to the next by a covalent bond and forms the backbone for the entire molecule. As described by Lawrence (1994) and Patel (1998) polymers offer a wide range of options about tuning the physicochemical properties. As a result the formulator got a finely tuned compound with desired quantities. As described by Porter (1994) polymers are used extensively in commercial sectors like textile industries, car industries and packaging industries. Polymers get an added value when these offers surfactant property. Nonionic surfactants are mostly polymers and these compounds usually form well distinct series. Pyrrolidones, polyoxyethylene glycols are two examples of such variety and may be called nonionic surfactant polymers (NISPOLs).

In pharmaceutical plants, NISPOLs are used as essential adjuvants for formulation of dosage forms. They stabilize multiphase preparations like dispersions (namely emulsions, suspensions and creams) and improve viscosity of water based formulations. Lievermann et al. (1990) and
Martin (1993) stated that these agents can architect new drug delivery systems. Structurally Pyrrolidones are synthetically produced homopolymer of $\mathrm{N}$-vinyl-2pyrrolidone with unique surfactant properties. Pyrrolidone $\mathrm{K}-30$ is a member of this series and this polymer being nonionic in nature exhibits low toxicity and higher biological compatibility and as such this was used as a plasma volume expander since 1930 (Login, 1995). According to Robinson (1990) this polymer is nonantigenic and requires no cross-matching and hence avoids the risk of infectious diseases such as HIV, hepatitis and typhoid associated with the cross-matching of blood.

Polyethylene glycols on the other hand consists of ethylene oxide monomers that are bonded with co-valent bond. The members of glycol series like PEG 3350, PEG 6000 and PEG 20000 are compounds of interest because of the versatile uses. Pashankar et al. (2003a \& b) and Pashankar et al. (2004) studied the efficacy of PEG 3350 in colonoscopy and chronic constipation in children. Moreover Baired et al. (2003) and Lukyanov et al. (2003) claimed that PEG 3350 retarded mechanical hemolysis, changed the inhibition pattern of immune response activation and as such was used in the treatment of tumor. In Pharmaceutical fields these two groups of NISPOLs play important roles in the solubilisation of drugs which

Correspondence to: Sakina Sultana; E-mail: sultana_s2003@yahoo.co.uk 
has limited water solubility. According to Joshi et al. (2004) these polymers are good solubilisers for a number of drugs including steroids. Besides NISPOLs are frequently used as binders and coating materials for tablets and viscosity enhancers for liquids. In fact the use of these nonionic surfactant polymers as viscosity enhancers has been well recognized since long time. Viscosity of a solution is a parameter that gives a measure of hydration in water. Water based formulations like suspensions, emulsions and ophthalmic solutions are preferred to be viscous as the viscous solution may minimize hindered settling of internal phase. This phenomenon protects the formulation from phase separation and increases product shelf life. Again a more viscous formulation prolongs drug residence time at the site of action and optimizes conditions for complete drug absorption. Therefore, selection of a proper viscosity enhancer is very important for drug stability and also for drug action. In practice viscosity of an agent is expressed as relative viscosity. But for polymers it is wise to express the parameter in terms of intrinsic viscosity which represents viscosity behavior of the agent without having solvent effect. Thus, in order to find out the viscosity property of polymers it is better to take intrinsic viscosity into consideration. Again in the viscosity measurements density is also important as it is directly involved with viscosity measurement according to Poiseuille's law. Our present work is therefore aimed at investigating the viscosity and density behavior of Pyrrolidone K-30, PEG 6000 and PEG 20000.

\section{Materials and Methods}

The work was conducted in the premises of Pharmacy Department of Jahangirnagar University, Savar, Dhaka, Bangladesh spanning a period of 3 months and the methods adopted for determining viscosity and density were as described by Patel (1998).

Viscosity measurement: In the method a 'Suspended level dilution viscometer' (immersed is a water bath maintained at room temperature $298 \pm 1{ }^{0} \mathrm{~K}$ ) was used. During the measurement serially diluted aqueous sample solutions (starting from a concentration of $4 \% \mathrm{w} / \mathrm{v}$ ) was filtered through a Whatman \#1 filter paper directly into the viscometer and allowed to equilibrate before any reading was made. Upon equilibration, time taken for solution to travel the distance between the defined two marks was recorded. The measurement was repeated on the same solution three times or until the flow time for three successive measurements agreed to within 1 second. The mean of the 3 readings was then used to calculate relative viscosity $\left(\mathrm{y}_{\mathrm{r}}\right)$ using filtered distilled water as a standard. In the study after each set of measurements, the sample solution was removed from the viscometer. Following a thorough rinse with distilled water the viscometer was completely dried out with methanol and the process was repeated with the next dilution. From $\mathrm{y}_{\mathrm{r}}$ the 'specific viscosity' $\left(\mathrm{y}_{\mathrm{sp}}\right.$ i.e., $\left.1-\mathrm{y}_{\mathrm{r}}\right)$ and 'reduced viscosity' ( $\mathrm{y}_{\mathrm{red}}$ i.e., $\mathrm{y}_{\mathrm{sp}} / \mathrm{c}$ ) were determined. These two later on gave a measure of viscosity of the polymer in terms of degree of hydration from the intercept of the plot of $\eta_{\text {red }}$ versus surfactant concentration at infinite dilution. In practice the intercept is known as 'intrinsic viscosity' and is denoted as [ $\mathrm{y}]$.

Density measurement: For determining density of the solutions, double distilled water filtered through a Whatman \#1 filter paper was directly poured into the clean, alcohol dried, pre-weighed density bottle to fill it completely. The bottle was then fitted with its stopper taking care not to introduce any air bubbles inside. Excess water coming out through the stopper was then wiped off carefully and the bottle was allowed to equilibrate at $298 \pm$ $1^{0} \mathrm{~K}$ for 10 minutes and then reweighed. Three successive readings were taken for each dilution and the mean was used for calculations. Absolute density of the sample was calculated from the line of best fit as obtained from the plot drawn with solution density versus concentration.

\section{Results and Discussion}

\section{Viscosity and density measurement}

Results of viscosity and density measurements have been shown in Table 1 along with Figures 1 and 2 .

The $\mathrm{y}_{\mathrm{r}}$ values of Pyrrolidone K-30 and PEGs as shown in table 1 state that the NISPOLs are viscous in nature and all of them undoubtedly had undergone extensive hydration. In general $[\mathrm{y}]$ or 'intrinsic viscosity' gives a measure of degree of hydration and a value greater than 10 shows an extensive hydration. In the study Pyrrolidone K30, PEG 6000 and PEG 20000 exhibited [y] value of $\sim 14$ $\mathrm{ml} / \mathrm{g}, 18 \mathrm{ml} / \mathrm{g}$ and $28 \mathrm{ml} / \mathrm{g}$, respectively (Figure 1). These results were similar to that obtained by Patel (1998). It has been reported earlier by Guner (1996) that Pyrrolidone K30 is a homopolymer of $N$-vinyl pyrrolidone and therefore 
the molecule possesses a pyrrole ring with a protonated nitrogen atom. Therefore, Pyrrolidone K-30 can act as a proton acceptor and can easily form hydrogen bond with water via the amide functions allowing water to enter and to solvate the molecule. It is therefore not surprising that Pyrrolidone K-30 would exhibit a considerable hydration although the molecule has hydrophobicity because of the presence of alkyl group in its structure. The case was similar with PEGs. PEGs got oxide linkage and these are also capable of forming hydrogen bond with water which made the molecule to be hydrophilic and the aqueous solution become viscous. In the study [ $\mathrm{\eta}$ ] values of PEGs were much higher than that of Pyrrolidone K-30 (Figure 1). Such difference in values may be due to the difference in molecular weight or may be due to polymer-solvent interaction. Usually in a poor solvent, polymer-polymerinteraction contacts become highly favored and as such monomer chain contacts resulting structural non-flexibility

Table 1. Relative viscosity and density values of aqueous solutions of Pyrrolidone K-30 (as donated by $\xi$ ), of PEG 6000 (as donated by $\psi$ ) and of PEG 20000 (as donated by $\varepsilon$ ).

\begin{tabular}{|c|c|c|c|c|}
\hline $\begin{array}{l}\text { Concentration } \\
(\mathrm{g} / \mathrm{ml})\end{array}$ & Density (g/cc) & Relative viscosity $\left(\mathrm{y}_{\mathrm{r}}\right)$ & $\begin{array}{l}\text { Specific viscosity } \\
\left(\mathrm{y}_{\mathrm{sp}}=1-\mathrm{y}_{\mathrm{r}}\right)\end{array}$ & $\begin{array}{c}\text { Reduced viscosity }\left(\mathrm{y}_{\mathrm{red}}=\right. \\
\mathrm{y}_{\mathrm{sp}} / \text { Conc.) i.e. } \mathrm{mL} / \mathrm{g}\end{array}$ \\
\hline \multirow[t]{2}{*}{0.005} & $0.9993^{\xi}$ & $1.0682^{\xi}$ & $0.0682^{\xi}$ & $13.6400^{\xi}$ \\
\hline & $0.9980^{\psi}$ & $1.0938 \psi$ & $0.0938 \psi$ & $18.7600 \psi$ \\
\hline \multirow[t]{3}{*}{0.010} & $1.0006^{\xi}$ & $1.1797^{\xi}$ & $0.1797^{\xi}$ & $17.9700^{\xi}$ \\
\hline & $0.9985^{\psi}$ & $1.1967^{\psi}$ & $0.1967^{\psi}$ & $19.6700^{\psi}$ \\
\hline & $1.0191 \varepsilon$ & $1.2931 \varepsilon$ & $0.2931 \varepsilon$ & $29.31 \varepsilon$ \\
\hline \multirow[t]{3}{*}{0.015} & $1.0017^{\xi}$ & $1.3070^{\xi}$ & $0.3070^{\xi}$ & $20.4667^{\xi}$ \\
\hline & $0.9994^{\psi}$ & $1.2955^{\psi}$ & $0.2955^{\psi}$ & $19.7800^{\psi}$ \\
\hline & $1.0195 \varepsilon$ & $1.4652 \varepsilon$ & $0.4652 \varepsilon$ & $31.0133 \varepsilon$ \\
\hline \multirow[t]{3}{*}{0.020} & $1.0030^{\xi}$ & $1.4664^{\xi}$ & $0.4664^{\xi}$ & $23.3200^{\xi}$ \\
\hline & $1.0003^{\psi}$ & $1.4351^{\psi}$ & $0.4351^{\psi}$ & $21.7550^{\psi}$ \\
\hline & $1.0199 \varepsilon$ & $1.6598 \varepsilon$ & $0.6598 \varepsilon$ & $32.99 \varepsilon$ \\
\hline \multirow[t]{3}{*}{0.030} & $1.0053^{\xi}$ & $1.7898^{\xi}$ & $0.7898^{\xi}$ & $26.3267^{\xi}$ \\
\hline & $1.0023^{\psi}$ & $1.7147^{\psi}$ & $0.7147^{\psi}$ & $23.8233^{\psi}$ \\
\hline & $1.0221 \varepsilon$ & $2.0208 \varepsilon$ & $1.0208 \varepsilon$ & $34.0267 \varepsilon$ \\
\hline \multirow[t]{3}{*}{0.040} & $1.0081^{\xi}$ & $2.1367^{\xi}$ & $1.1367^{\xi}$ & $28.4175^{\xi}$ \\
\hline & $1.0040^{\psi}$ & $1.9860^{\psi}$ & $0.9860^{\psi}$ & $24.6467^{\psi}$ \\
\hline & $1.0277 \varepsilon$ & $2.4169 \varepsilon$ & $1.4169 \varepsilon$ & $35.4225 \varepsilon$ \\
\hline
\end{tabular}

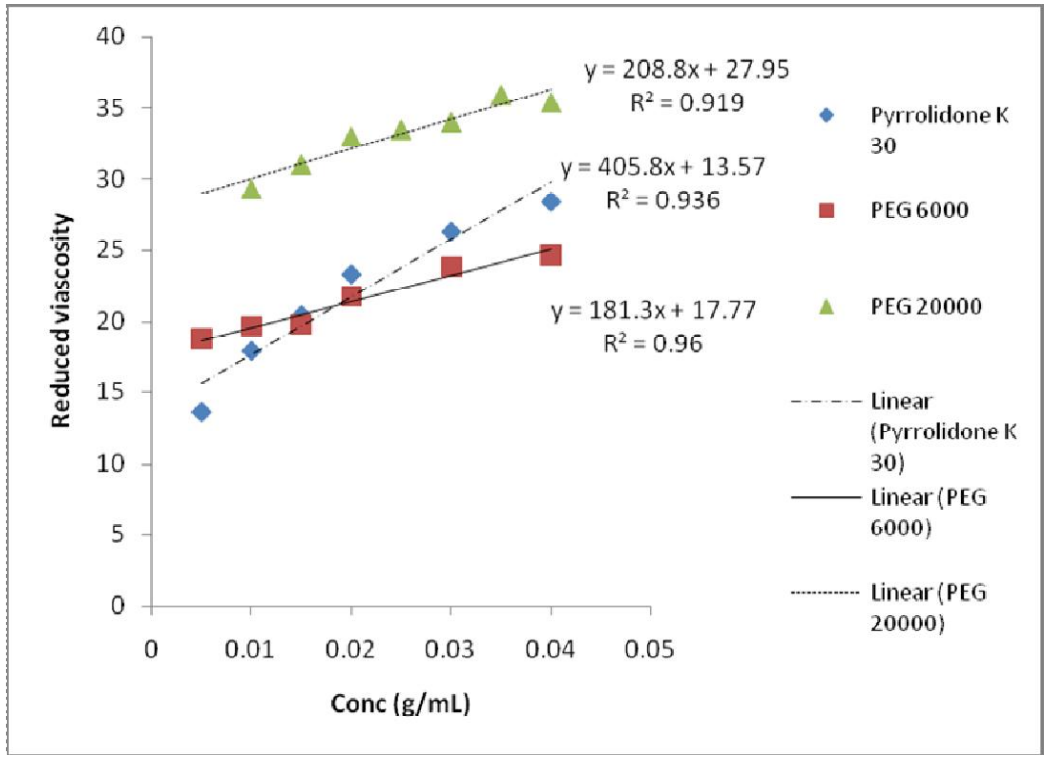

Figure 1. Intrinsic viscosity of Pyrrolidone K-30, PEG 6000 \& PEG 20000. 


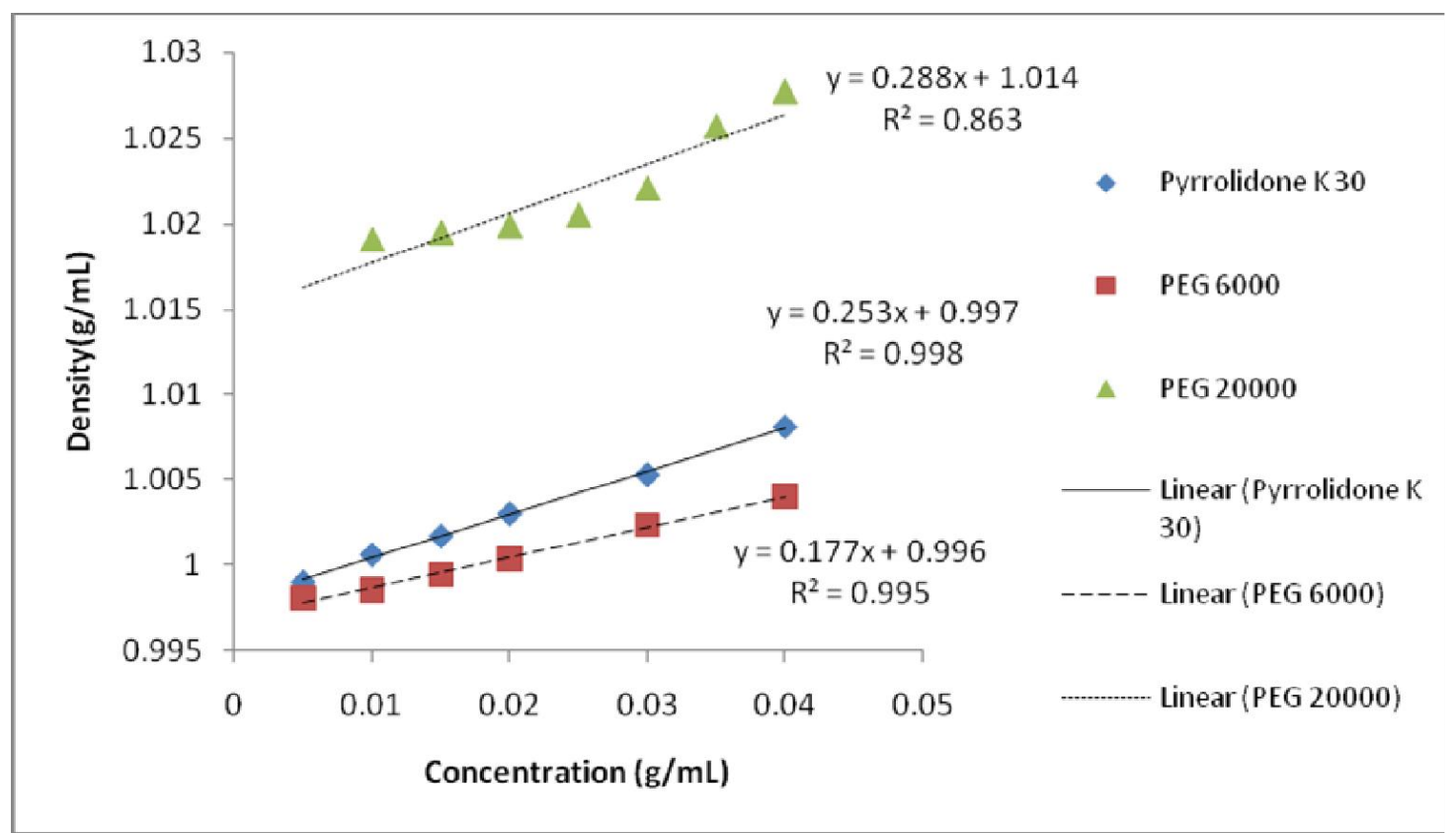

Figure 2. Density parameters of Pyrrolidone K-30, PEG 6000 \& PEG 20000.

that prevents penetration of solvent molecule into the polymer conformation. As a consequence polymer molecule becomes less solvated. The phenomenon reverses with a good solvent. In the present study water was used and it is a good solvent. Yet there was difference in the extent of hydration between these polymers. It is presumed that Pyrrolidone K-30 has longer chain favoring more polymer-polymer contact sites upon coiling. Therefore, it was not unlikely that Pyrrolidone K30 has more restricted excluded volume and takes a nonflexible conformation compared to PEGs as described by Attwood and Fluorence (1983), Saito and Sato (1985) and Guner and Kara (1998). However, in the present study between PEG 6000 and PEG 20000 the intrinsic viscosity of PEG 6000 was found much lower than PEG 20000, which may not be the usual case. Usually, viscosity of a compound is related to its molecular weight. Here molecular weight of PEG 20000 was higher than PEG 6000 , yet it showed higher intrinsic viscosity than PEG 6000 meaning higher tendency to be hydrated is not in the line of general agreement. This anomaly can be explained by the fact that as a polymeric molecule like PEG 20000 had a higher number of glycol units and this higher number had imparted the molecules a higher tendency to be hydrated as glycols tends to be polar yet this needs further study to confirm. In the density study Pyrrolidone K-30, PEG 6000 and PEG 20000 showed absolute density slightly higher than unity (Figure 2). Results respective to PEGs are in accordance with that as published in Apha (1986). In our study, the trend of change in density with the variations in surfactant concentration was investigated. For Pyrrolidone K-30 and PEG 6000 the trend was linear but for PEG 20000 the trend was non-linear. Such discrepancy may be related to non-newtonian flow property of the compound. Therefore studies are needed to get a more comprehensive and clear picture about the hydration and density of the studied polymers.

\section{References}

A. PhA. 1986. American Pharmaceutical Association and the Pharmaceutical Society of Great Britain. Handbook of Pharmaceutical Excipients. pp. 209-234.

Attwood, D. and Florence, A.T. 1983. Surfactant systems-their Chemistry, Pharmacy and Biology. Chapman \& Hall Publication, London.

Baired, E.J., Holowka, D., Coates, G.W and Baird, B. 2003. Highly effective poly architectures for specific immune receptor activation. Biochemistry 42, 12739-12748.

Guner, A. and Kara, M. 1998. Cloud points and temperatures of aqueous polyvinyl pyrrolidone solutions in the presence of denaturing agents. Polymers 39, 1569-1572. 
Guner, A. 1996. Properties of aqueous salt solutions of polyvinyl pyrrolidone. J. Apple Polymer. Sci. 62, 785-788.

Joshi, H.N., Tejwan, R.W., Davidivich, M., Jemal, M., Sahashrabudhe, V.P., Bathala, M.S., Varia, S.A. and Serajuddin, A.T. 2004. Bioavailability enhancement of a poorly water soluble drug by solid dispersion in polyehhylene glycol-polysorbate mixture. Int. J. Pharm. 269, 251-258.

Laurence, M.J. 1994. Surfactant system: their use in drug delivery. Chem. Soc. Rev. 417-423.

Lievermann, H.A., Lachman, L. and Schwartz, J.B. 1990. Pharmaceutical dosage forms. $1^{\text {st }}$ Edition. Marcel Dekker Inc. New York.

Login, R.B. 1995. Pyrrolidone-base surfactants: a literature review. JAOCS. 72, 759-771.

Lukyanov, A.N., Gao, Z and Torchilin, V.P. 2003. Micelles from polyethelene glycol/phosphatidylethanolamine conjugates for tumor drug delivery. J. Control. Release 1-2. 97-102.

Martin, A. 1993. Physical Pharmacy. Fourth edition. Waverly International, Maryland, USA. 557-567.

Pashankar, D.S., Bishop, W.P and Loening-Baucke, V. 2003a. Long term efficacy of Polyethylene glycol for the treatment of chronic constipation in children with or without encopresis. J. Clin. Pediatr. 42, 815-819.
Pashankar, D.S., Bishop, W.P and Loening-Baucke, V. 2003b. Safety of polyethylene glycol 3350 for the treatment of chronic constipation in children. Arch. Pediatr. Adolese. Med. 157, 661-664.

Pashankar, D.S., UC, A. and Bishop, W.P. 2004. Polyethylene glycol 3350 without electrolyte: a new safe, effective and palatable bowel preparation for colonscopy in children. $J$. Pediatr. 144, 358-362.

Patel, R. 1998. Novel Polyethylene pyrrolidone-based surfactants: their synthesis and Physico-chemical properties. Ph.D. thesis, University of London, London.

Porter, M.R. 1994. Handbook of Surfactants. $1^{\text {st }}$ edition. Blackie Academic and Professional- Chapman \& Hall, London.

Robinson, B.V., Sullivan, F.M. and Schwartz, S.L. 1990. PVP-a critical review of the kinetics and toxicology of polyvinylpyrrolidone (Povidone). Lewis Publishers Inc., Michigan.

Saito, Y. and Sato, T. 1985. Effects of polyoxyethylene chain length on micellar structure. J. Phys. Chem. 89, 2110-2113. 